\title{
Pharmacological activation of a novel p53-dependent S-phase checkpoint involving CHK-1
}

\author{
A Ahmed ${ }^{1}$, J Yang ${ }^{1,3}$, A Maya-Mendoza ${ }^{2}$, DA Jackson ${ }^{2}$ and M Ashcroft ${ }^{\star, 1}$
}

We have recently shown that induction of the p53 tumour suppressor protein by the small-molecule RITA (reactivation of p53 and induction of tumour cell apoptosis; 2,5-bis(5-hydroxymethyl-2-thienyl)furan) inhibits hypoxia-inducible factor-1 $\alpha$ and vascular endothelial growth factor expression in vivo and induces p53-dependent tumour cell apoptosis in normoxia and hypoxia. Here, we demonstrate that RITA activates the canonical ataxia telangiectasia mutated/ataxia telangiectasia and Rad3related DNA damage response pathway. Interestingly, phosphorylation of checkpoint kinase (CHK)-1 induced in response to RITA was influenced by p53 status. We found that induction of p53, phosphorylated CHK-1 and $\gamma \mathrm{H} 2 \mathrm{AX}$ proteins was significantly increased in S-phase. Furthermore, we found that RITA stalled replication fork elongation, prolonged S-phase progression and induced DNA damage in p53 positive cells. Although CHK-1 knockdown did not significantly affect p53-dependent DNA damage or apoptosis induced by RITA, it did block the ability for DNA integrity to be maintained during the immediate response to RITA. These data reveal the existence of a novel p53-dependent S-phase DNA maintenance checkpoint involving CHK-1.

Cell Death and Disease (2011) 2, e160; doi:10.1038/cddis.2011.42; published online 19 May 2011

Subject Category: Cancer

Solid tumours characteristically contain areas of low oxygen tension (hypoxia). Hypoxia stabilises the expression of the hypoxia-inducible factor (HIF)- $\alpha$ transcription factor. Deregulated overexpression of HIF- $\alpha$ in tumour cells initiates a transcriptional programme that renders tumour cells resistant to chemotherapy and radiotherapy, resulting in a more aggressive and metastatic cancer phenotype. ${ }^{1}$ Targeting $\mathrm{HIF} /$ hypoxia signalling, therefore, has become an attractive strategy for the development of new cancer treatments. ${ }^{2}$

The p53 tumour suppressor protein is a potent negative regulator of HIF- $1 \alpha$, mediating both apoptotic ${ }^{3,4}$ and antiangiogenic effects when overexpressed. ${ }^{5,6} \mathrm{HIF}-1 \alpha$ accumulation in hypoxia is blocked by overexpression ${ }^{5}$ or activation ${ }^{6}$ of p53, and HIF-1-dependent transcription negatively correlates with p53 status. ${ }^{7}$ p53 is mutated in about $50 \%$ of human cancers, and several agents have been described that can reactivate mutant ${ }^{8,9}$ or activate wild-type $p 53^{10-12}$ in tumour cells. However, many of these emerging p53-targeted agents have not yet been evaluated for their effectiveness at mediating tumour cell death in normoxia and hypoxia. We have been exploring the mechanistic properties of the smallmolecule activator of p53, RITA (reactivation of p53 and induction of tumour cell apoptosis; 2,5-bis (5-hydroxymethyl2-thienyl) furan, NSC-652287). ${ }^{12-14}$ RITA was originally identified in a cell-based screen using the National Cancer Institute compound library and was shown to mediate p53- dependent antitumour activity in vivo. ${ }^{12}$ Subsequently, we have found that RITA significantly inhibits HIF- $1 \alpha$ induction and elicits p53-dependent apoptotic responses in normoxia and hypoxia, and promotes both apoptotic and antiangiogenic effects in vivo. ${ }^{15}$ RITA was originally proposed to stabilise and activate $\mathrm{p} 53$ by disruption of the p53-human double minute 2 (HDM2) interaction, ${ }^{12}$ and mediate p53-dependent apoptosis and other cellular responses via the regulation of p53 transcriptional responses. ${ }^{16}$ Recently, we have shown that RITA mediates an effect on the protein translation machinery and downregulates both HDM2 and p21 protein levels in a dose- and time-dependent manner while concurrently inducing apoptosis. ${ }^{15}$ Moreover, and consistent with previous reports indicating that RITA causes protein-DNA and DNADNA intrastrand crosslinks, ${ }^{13,14}$ we have found that RITA activates a DNA damage response. ${ }^{17}$ Collectively, these studies indicate that RITA exhibits a complex mechanism of action that leads to p53 activation and apoptotic responses.

Here, we further explore the mechanism of action of RITA, with particular focus on the DNA damage response. We demonstrate that RITA activates the canonical ataxia telangiectasia mutated/ataxia telangiectasia and Rad3-related (ATM/ATR) DNA damage cascade and induces DNA damage in p53 positive cells. Interestingly, RITA also mediates checkpoint kinase (CHK)-1 phosphorylation and slows replication fork elongation and S-phase progression in a

\footnotetext{
${ }^{1}$ Department of Metabolism and Experimental Therapeutics, Division of Medicine, Centre for Cell Signalling and Molecular Genetics, University College London, Rayne Building, 5 University Street, London WC1E 6JJ, UK and ${ }^{2}$ Faculty of Life Sciences, University of Manchester, MIB, Manchester, M1 7DN, UK

${ }^{*}$ Corresponding author: M Ashcroft, Department of Metabolism and Experimental Therapeutics, Division of Medicine, Centre for Cell Signalling and Molecular Genetics, University College London, Rayne Building, 5 University Street, London WC1E 6JJ, UK. Tel: + 44207679 0799; Fax: + 442076796211 ;

E-mail: m.ashcroft@ucl.ac.uk

${ }^{3}$ Current address: Department of Genetics and Tumour Cell Biology, St. Jude Children's Research Hospital, 262 Danny Thomas Place, Memphis, TN 38105, USA Keywords: p53; hypoxia; HIF-1 $\alpha$; DNA damage; CHK-1

Abbreviations: RITA, reactivation of p53 and induction of tumour cell apoptosis; HIF-1 $\alpha$, hypoxia-inducible factor- $1 \alpha$; ATM, ataxia telangiectasia mutated; ATR, ataxia telangiectasia and Rad3 related; CHK, checkpoint kinase; HDM2, human double minute 2; PARP, poly ADP ribose polymerase; BrdU, bromodeoxyuridine

Received 08.4.11; accepted 11.4.11; Edited by G Melino
} 
p53-dependent manner. Loss of CHK-1 does not significantly affect p53-mediated apoptotic responses induced by RITA, but does significantly enhance DNA damage upon short term exposure to RITA. Our study identifies a novel p53-dependent S-phase checkpoint involving CHK-1.

\section{Results}

RITA induces a DNA damage response. Recently, we have found that RITA can mediate significant tumour cell apoptosis in normoxia and hypoxia in a p53-dependent manner and activate a DNA damage response in vitro and in vivo. ${ }^{15}$ Consistent with our previous studies, ${ }^{17}$ here, we found that RITA induced phosphorylation of the histone protein $\mathrm{H} 2 \mathrm{AX}$ at Ser139 $((\gamma \mathrm{H} 2 \mathrm{AX})$; Figure $1 \mathrm{a})$, which is usually associated with DNA damage-induced stress. ${ }^{18}$ Concurrently, we found that RITA increased cleaved poly ADP ribose polymerase (PARP; Figure 1a) and the percentage of cells in sub-G1 (Figure 1b), indicative of increased apoptosis. Both DNA damage and apoptotic responses induced by RITA were observed in normoxia and hypoxia, only in p53-positive cells (Figure 1).

To further explore the DNA damage response induced by RITA, we first assessed the phosphorylation status of p53
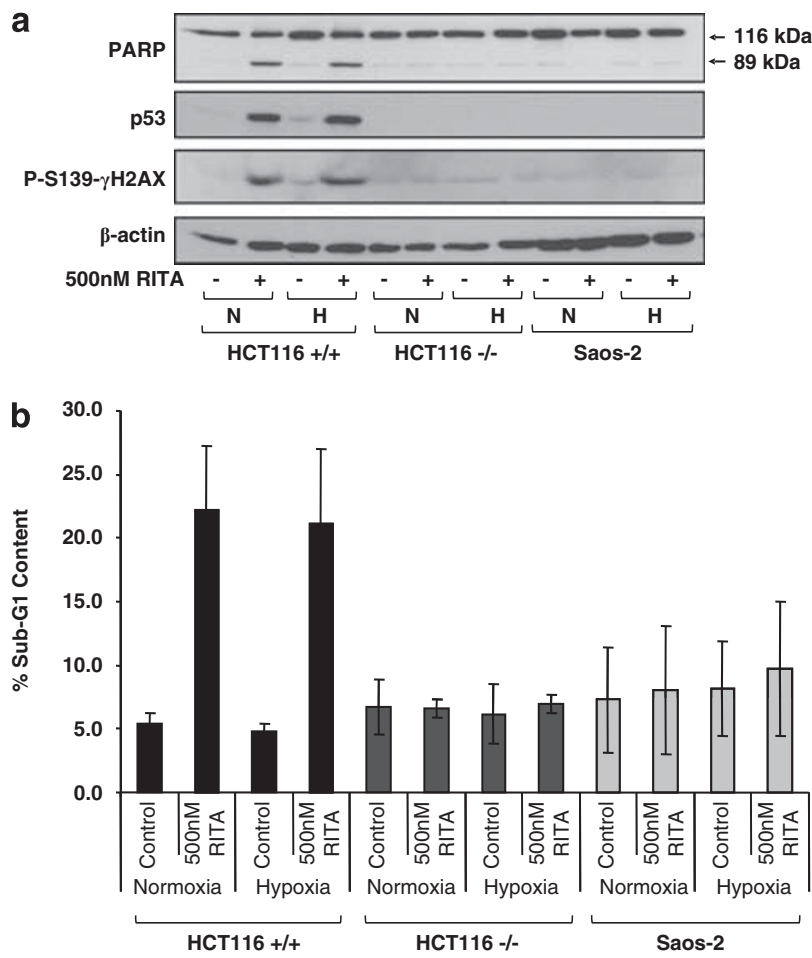

Figure 1 RITA induces p53-dependent apoptosis in normoxia and hypoxia. (a and b) p53 $3^{-l-}$ HCT116, p53 $3^{+/+}$HCT116 cells or Saos-2 (p53 null) cells were treated with or without RITA $(500 \mathrm{nM})$ for $16 \mathrm{~h}$ in either normoxia or hypoxia $\left(1 \% \mathrm{O}_{2}\right)$. (a) Western blots show cleaved PARP $(89 \mathrm{kDa})$, p53 and phosphorylated-S139$\gamma \mathrm{H} 2 \mathrm{AX}$ proteins. Actin was used as a loading control. (b) In parallel with the western analysis described in a, cells were harvested for flow cytometric analysis. Cells were fixed and stained using propidium iodide to visualise DNA profiles. The graph shows the percentage (\%) of cells in sub-G1 in response to the treatments as indicated. Data is averaged from three independent experiments in response to RITA, as phosphorylation of p53 within the $\mathrm{N}$-terminus is usually induced by genotoxic stress. ${ }^{19} \mathrm{p} 53^{+1+}$ HCT116 cells were treated with RITA over a concentration curve and assessed for Ser15, Ser20 and Ser46 phosphorylation of p53 (Figure 2a). We found that RITA induced $\mathrm{N}$-terminal phosphorylation of p53 (Figure 2a). DNA damage is usually sensed by the PI-3K-related protein kinases ATM and ATR, which activate the transducer checkpoint kinases CHK-2 and CHK-1, respectively. ${ }^{20}$ ATM/ATR directly phosphorylates p53 at Ser15, whereas CHK-2/CHK-1 phosphorylates Ser20 on p53. ${ }^{21}$ Consistent with activation of the canonical ATM/ATR DNA damage response pathway, we found that RITA also induced phosphorylation of Ser345 on CHK-1 and Thr68 on CHK-2 (Figure 2a), and increased $\gamma \mathrm{H} 2 \mathrm{AX}$ in a dose-dependent manner (Figure 2a). These responses correlated with a dose-dependent increase of cells in sub-G1, indicative of apoptosis (Figure 2a, graph). In addition, we found that induction of phosphorylated $\mathrm{CHK}-1$, CHK-2, p53 and $\gamma \mathrm{H} 2 \mathrm{AX}$ proteins was time-dependent (Figures $2 \mathrm{~b}$ and $\mathrm{c}$ ) and correlated with a time-dependent increase in cells in sub-G1 (Figure 2d). Thus, the DNA damage response induced by RITA is both dose- and time-dependent.

We found that the induction of phosphorylated p53 (Ser15 and Ser20), CHK-1 and CHK-2 proteins in response to RITA was blocked by wortmannin, a kinase inhibitor of ATM/ATR and other phosphatidylinositol 3-kinase family members (Figure 2e), whereas the induction of cleaved PARP was only marginally affected (Figures $2 e$ and f). Interestingly, we found that wortmannin had no significant effect on the induction of eukaryotic initiation factor- $2 \alpha$ phosphorylation or the downregulation of HDM2 and p21 proteins induced by RITA (Figure 2f), which we have previously described. ${ }^{15}$ These data indicate that the DNA damage and translational responses induced by RITA are potentially separable processes. As we would anticipate from the wortmannin effects observed (Figures $2 e$ and $f$ ), in response to RITA, we found that ATM or ATR siRNA blocked the induction of phosphorylated CHK-1 and CHK-2 (Figure 2g), which are downstream targets of ATR and ATM, respectively. Finally, further analysis of the DNA damage response induced by RITA indicated a slight but measurable increase in DNA damage in p53-positive HCT116 and MCF-7 cells (Figures $3 a$ and b), which was not observed in p53-null HCT116 or Saos-2 cells (Figure $3 \mathrm{c}$ ). Taken together, these data suggest that RITA activates the canonical ATM/ATR DNA damage response pathway and induces DNA damage in p53 positive cells.

RITA stalls replication fork elongation and prolongs S-phase progression in p53-positive cells. We have previously observed that most cells treated with RITA showed an intense pan-nuclear staining of $\gamma \mathrm{H} 2 \mathrm{AX} .{ }^{17} \mathrm{~A}$ similar pan-nuclear staining pattern was observed in response to hydroxyurea treatment. ${ }^{17,22}$ This type of DNA damage response is indicative of potential stalling of the replication fork or inhibition of replication fork elongation, and can be mediated during processing of bulky DNA lesions. ${ }^{22}$ During replication, cells usually respond to DNA damage by 
a
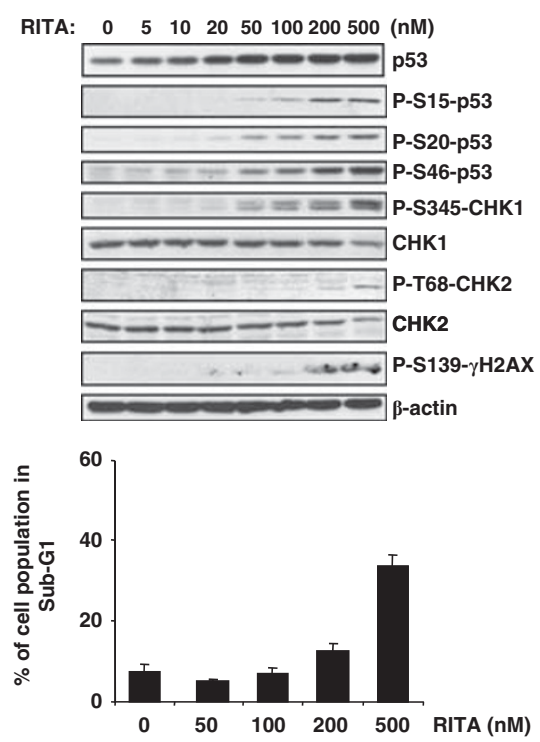

$f$ RITA: - + - +

\section{b}

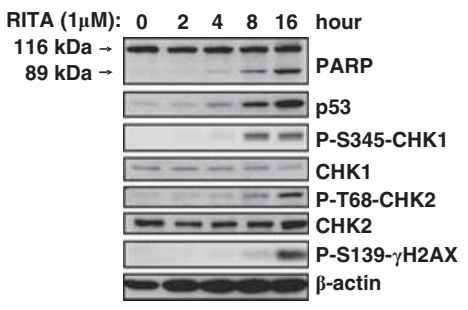

d

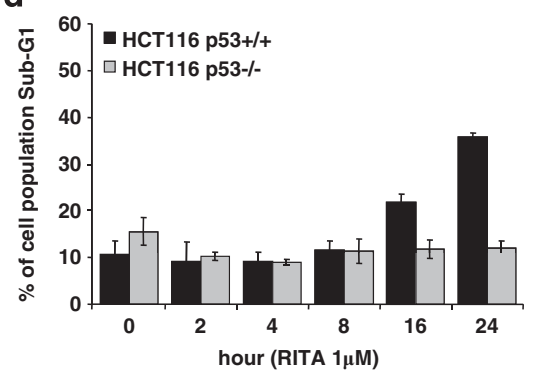

C

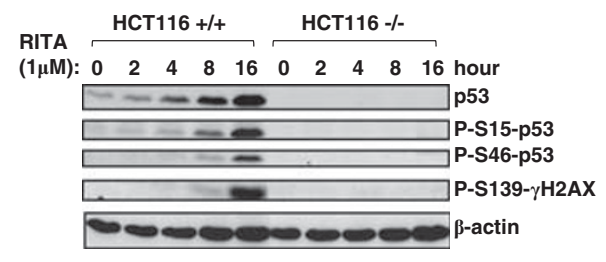

e

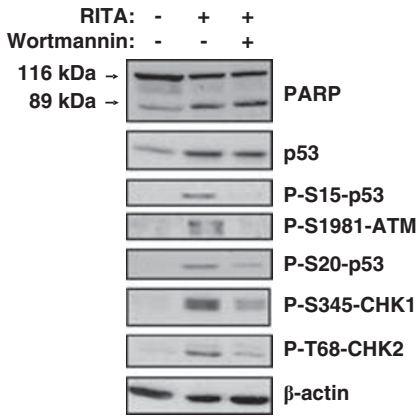
Wortmannin: --++
$116 \mathrm{kDa} \rightarrow-$ - PARP $89 \mathrm{kDa}-$

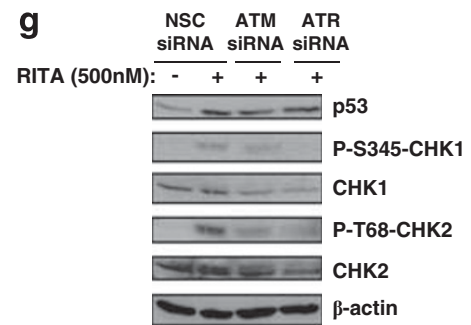

Figure 2 RITA induces a DNA damage response. (a) Western blot analysis of DNA damage response markers in $553^{+1+} \mathrm{HCT} 116$ cells treated with the indicated concentrations of RITA for $16 \mathrm{~h}$. Western blot analysis shows p53, phosphorylated p53 (S15, S20, S46), phosphorylated-S345-CHK-1, phosphorylated-T68-CHK-2, phosphorylated-S139- $\gamma \mathrm{H} 2 \mathrm{AX}, \mathrm{CHK}-1$ and CHK-2 proteins. Actin was used as a loading control. In parallel, cells were harvested for flow cytometric analysis. Cells were fixed and stained using propidium iodide to visualise DNA profiles. The graph shows the percentage (\%) of cells in sub-G1 in response to RITA treatment as indicated. Data is averaged from three independent experiments. (b) Western blot analysis of DNA damage response markers in $\mathrm{p} 53^{+1+} \mathrm{HCT} 116$ cells treated with RITA (1 $\left.\mu \mathrm{M}\right)$ for the indicated time. Western blot analysis shows p53, phosphorylated-S345-CHK-1, phosphorylated-T68-CHK-2, phosphorylated-S139- $\mathrm{H} 2 \mathrm{AX}$, CHK-1 and CHK-2 proteins Actin was used as a loading control. (c) Western blot analysis shows p53, phosphorylated p53 (S15 and S46) and phosphorylated-S139- $\gamma \mathrm{H} 2 \mathrm{AX}$ in p53 ${ }^{+/+} \mathrm{HCT} 116$ cells and p53 ${ }^{-1}$ ${ }^{-}$HCT116 cells treated with RITA $(1 \mu \mathrm{M})$ for the indicated time. Actin was used as a loading control. (d) In parallel with the western analysis described in c, cells were harvested for flow cytometric analysis. Cells were fixed and stained using propidium iodide to visualise DNA profiles. The graph shows the percentage (\%) of cells in sub-G1 in response to RITA treatment as indicated. Data are averaged from three independent experiments. (e and f) $p 53^{+1+}$ HCT116 cells were treated with RITA $(1 \mu \mathrm{M})$ in the presence or absence of wortmannin $(10 \mu \mathrm{M})$ for $16 \mathrm{~h}$ and assessed by western blot for p53, phosphorylated p53 (S15 and S20), phosphorylated-S1981-ATM, phosphorylated-S345-CHK1, phosphorylated-T68-CHK-2 proteins, HDM2, p21, phosphorylated eukaryotic initiation factor-2 $\alpha$ (elF-2 $\alpha$ ), total elF-2 $\alpha$, and cleaved PARP proteins. Actin was used as a loading control. (g) p53 ${ }^{+1+}$ HCT116 cells were transfected with a non-silencing control siRNA (NSC), ATM siRNA or ATR siRNA. Cells were treated with RITA (500 nM) for $24 \mathrm{~h}$ then harvested for western analysis. Western blot analysis shows p53, phosphorylated-S345-CHK-1, phosphorylated-T68-CHK-2, CHK-1 and CHK-2 proteins. Actin was used as a loading control

activating an intra-S-phase checkpoint. ${ }^{23}$ Therefore, we next assessed whether RITA mediated a p53-dependent DNA damage response by affecting the replication fork and S-phase progression. To do this, we treated $\mathrm{p} 53^{-1-}$ or p53 ${ }^{+/+}$HCT116 cells with RITA and performed a DNA fibre assay, as previously described. ${ }^{24,25}$ We found that RITA induced a marked increase in the percentage of replication

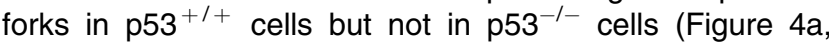
compare $\mathrm{p} 53^{-1-}$ with $\mathrm{p} 53^{+1+}$, lanes 2 and 3 ). Intriguingly, this effect was only observed in a sub-population of replicons, such that the second and third classes were very substantially affected by RITA treatment, whereas the seventh/eight/ninth classes were less affected (Figure $4 \mathrm{a}$,

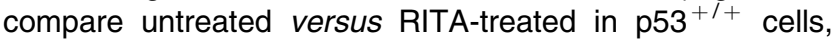

lanes 2 and 3 with lanes 7, 8 and 9). Statistical analysis ( $t$-test) showed significance $(P$-value $=4.85 \mathrm{E}-25)$ in $\mathrm{p} 3^{+1+}$ HCT116 cells for untreated versus RITA-treated (Figure $4 b$ ). Visualisation of DNA foci using bromodeoxyuridine (BrdU) pulse labelling and immunohistochemical analyses was performed to assess S-phase progression in unsynchronised $\mathrm{p} 53^{-1-}$ and $\mathrm{p} 53^{+1+} \mathrm{HCT} 116$ cells. We found that although the S-phase programme was maintained upon treatment with RITA, our data indicated that S-phase was prolonged at mid-late stages (Figure 4c).

Previous studies have shown that $\mathrm{CHK}-1$ predominantly regulates DNA replication, fork elongation and effects S-phase progression. ${ }^{25}$ As we found that RITA induced a p53-dependent increase in replication fork number 
a
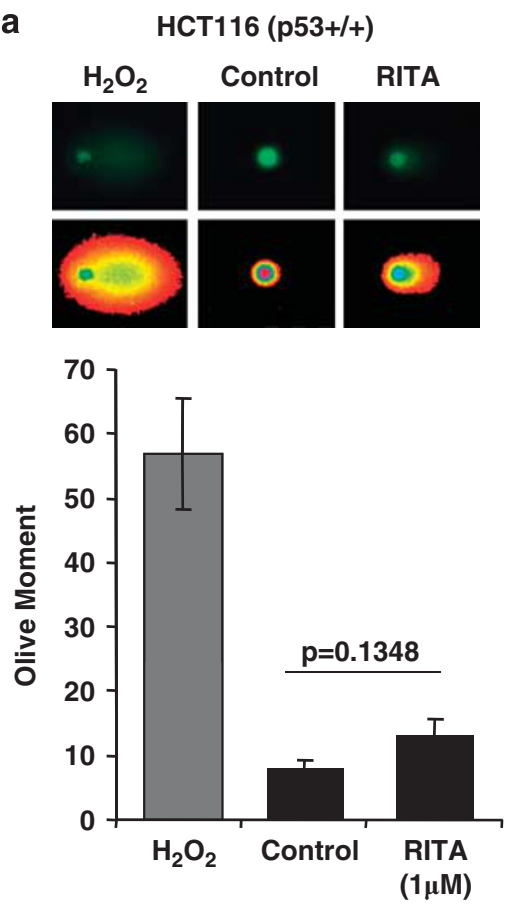

b
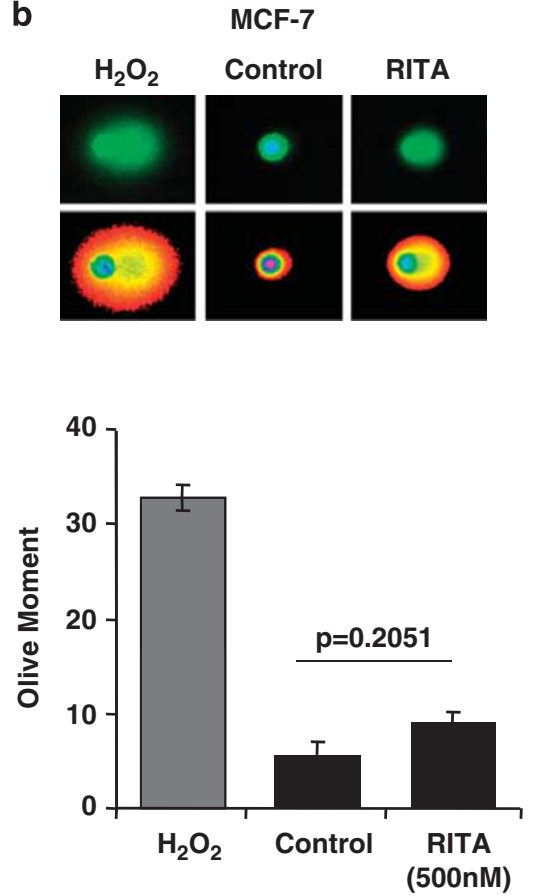

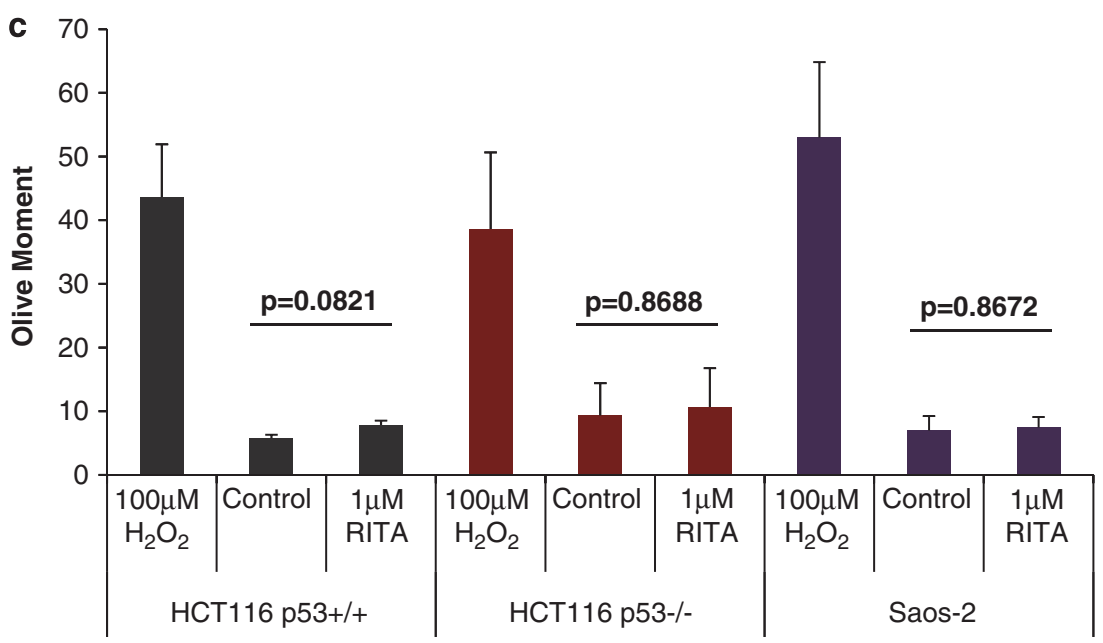

Figure 3 RITA induces DNA damage in p53-positive cells. (a) p53 ${ }^{+/+}$HCT116 and (b) MCF-7 cells were treated with RITA at the indicated concentrations and assessed for DNA strand breaks using the comet assay (upper panels show representative Sybr green-stained comets (upper row) and the respective digitally converted images (lower row) for each condition as indicated). As a positive control for DNA damage, cells were treated with $\mathrm{H}_{2} \mathrm{O}_{2}$ (100 $\mu \mathrm{M}$ for 20 min). Olive moment for each condition ( 100 comets/ sample) was measured using the Comet Score software (TriTek Corporation, Sumerduck, VA, USA). Graphs (lower panels) show mean olive moment as indicated. (c) p53 ${ }^{+1}$ ${ }^{+}$HCT116 p53 ${ }^{-1}$ HCT116 and Saos-2 were treated as described in a. Graph shows average olive moment as a measurement of DNA damage. Data is represented from three independent experiments

(Figures $4 \mathrm{a}$ and $\mathrm{b}$ ) and affected S-phase progression (Figure 4c), we next assessed whether CHK-1 phosphorylation was also affected by $\mathrm{p} 53$ status. To do this, $\mathrm{p} 53^{-1-}$ and p53 ${ }^{+1+}$ HCT116 cells were treated with RITA. We found that both $\mathrm{CHK}-1$ and $\mathrm{CHK}-2$ were phosphorylated in response to RITA treatment (Figure 4d). However, we found that phosphorylation of CHK-1 at Ser345 induced by RITA was affected by $\mathrm{p} 53$ status, whereas RITA-induced phosphorylated CHK-2 was observed in both $\mathrm{p} 53^{-1-}$ and $\mathrm{p}^{2} 3^{+/+}$cells to a similar extent (Figure 4d). Taken together, our studies indicate that RITA activates a p53-dependent DNA damage response involving $\mathrm{CHK}-1$ that functions to stall DNA replication fork elongation and prolong S-phase progression.

RITA induces p53 and phosphorylated CHK-1 and $\gamma \mathrm{H} 2 \mathrm{AX}$ proteins in S-phase. Given that RITA induces significant p53-dependent apoptotic (Figure 1) and S-phase responses (Figure 4), we next explored the induction of p53 and $\gamma \mathrm{H} 2 \mathrm{AX}$ proteins in sub-G1 and S-phase cell populations using fluorescence-activated cell sorting (FACS) analysis 
a
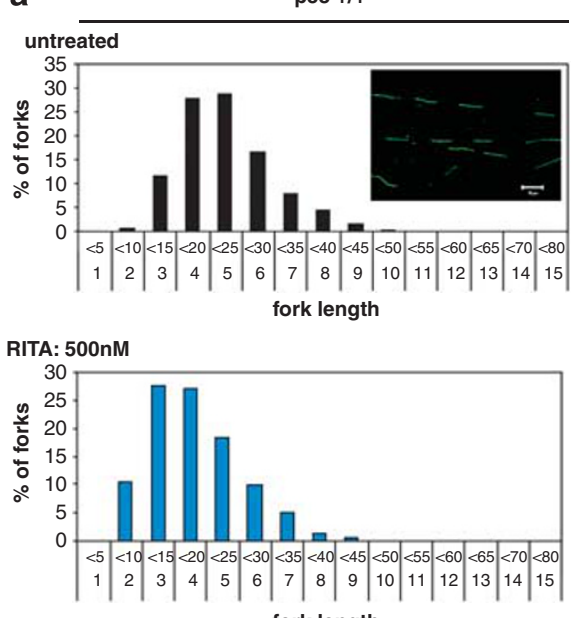

p53 -/

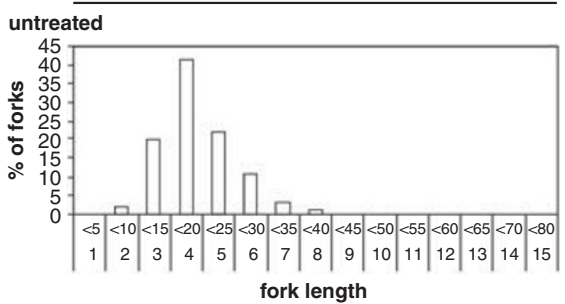

RITA: 500nM

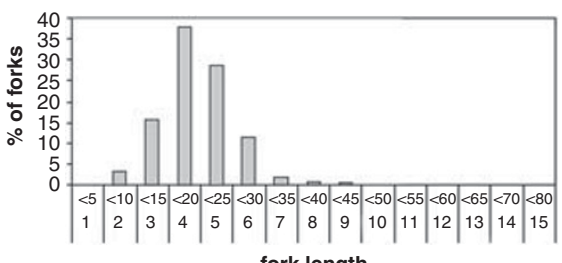

fork length

b

\begin{tabular}{|c|c|c|c|}
\hline Cell line & $\begin{array}{c}\text { Kbp/min } \\
\text { Mean }\end{array}$ & SD & $\mathbf{n}$ \\
\hline p53+/+ untreated & 1.125 & 0.34 & 601 \\
\hline $\begin{array}{c}\text { p53+/+ } \\
\text { RITA: 500nM }\end{array}$ & 0.9 & 0.34 & 551 \\
\hline $\begin{array}{c}\text { p53-/- } \\
\text { untreated }\end{array}$ & 0.95 & 0.26 & 577 \\
\hline $\begin{array}{c}\text { p53-/- } \\
\text { RITA: 500nM }\end{array}$ & 0.97 & 0.26 & 300 \\
\hline
\end{tabular}
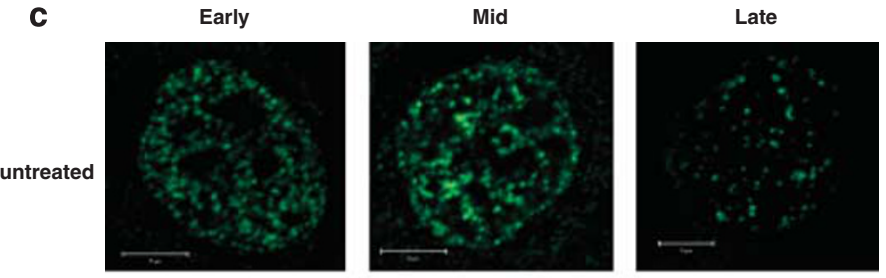

d
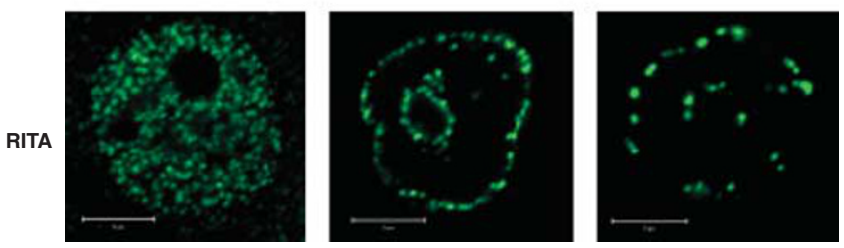

Alexa-488

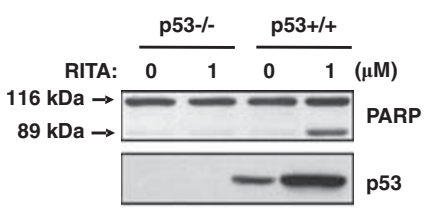

Alexa-488

Figure 4 RITA stalls replication fork elongation and slows S-phase progression in p53-positive cells. (a) DNA fibre assay of p53 $3^{-1-}$ and p53 ${ }^{+/+} \mathrm{HCT} 116 \mathrm{cells}$ treated with RITA $(500 \mathrm{nM})$ for $16 \mathrm{~h}$. Graphs show percentage (\%) number of forks versus fork length for each condition as indication. (b) Table (lower panel) shows a summary of the results from a for each condition analysed. Statistical analysis $\left(t\right.$-test) showed significance $(P$-value $=4.85 \mathrm{E}-25)$ for untreated versus RITA treated in p53 ${ }^{+/+}$HCT116 cells. (c) RITA prolongs S-phase in p53 ${ }^{+/+} \mathrm{HCT} 116$ cells. Immunofluorescence analysis shows DNA replication foci (Alexa-488, green) in p53 ${ }^{+/+} \mathrm{HCT} 116$ cells after treatment with RITA (500 nM) for $16 \mathrm{~h}$. BrdU incorporation was used as a measure for S-phase progression as indicated (early, mid and late stages). (d) Western blot analysis shows phosphorylated-S345-CHK-1, phosphorylated-T68-CHK-2, phosphorylated-S139- $\gamma$ H2AX and PARP proteins in p53 $3^{-I}$ and p53 ${ }^{+/+}$HCT116 cells treated with RITA (1 $\mu$ M) for $16 \mathrm{~h}$. Actin was used as a loading control

(Figure 5a). We found that p53 protein was induced in response to RITA in all cell cycle phases $(\mathrm{G} 1=38 \%$, $\mathrm{S}=43 \%$ and $\mathrm{G} 2=40 \%$ ). Interestingly, after treatment with RITA, a higher proportion of cells in S-phase were positive for $\gamma \mathrm{H} 2 \mathrm{AX}$ protein $(\mathrm{G} 1=22 \%, \mathrm{~S}=26 \%$ and $\mathrm{G} 2=10 \%$ ). Moreover, we found a dose- and time-dependent increase in both $\mathrm{p} 53$ and $\gamma \mathrm{H} 2 \mathrm{AX}$ protein levels in cells in S-phase in response to RITA (Figures $5 b$ and $c$ ). In addition, we found a significant increase in the proportion of cells in S-phase expressing phosphorylated $\mathrm{CHK}-1$ protein (Figures 5d and e), whereas an increase in cells expressing phosphorylated $\mathrm{CHK}-1$ protein was not observed in cells in sub-G1 (data not shown). Together, these data suggest that RITA activates a p53-dependent S-phase checkpoint involving $\mathrm{CHK}-1$.

CHK-1 knockdown does not affect RITA-induced apoptosis but affects DNA damage in cells treated with RITA. To further assess whether CHK-1 was required for p53-dependent apoptotic and DNA damage responses 

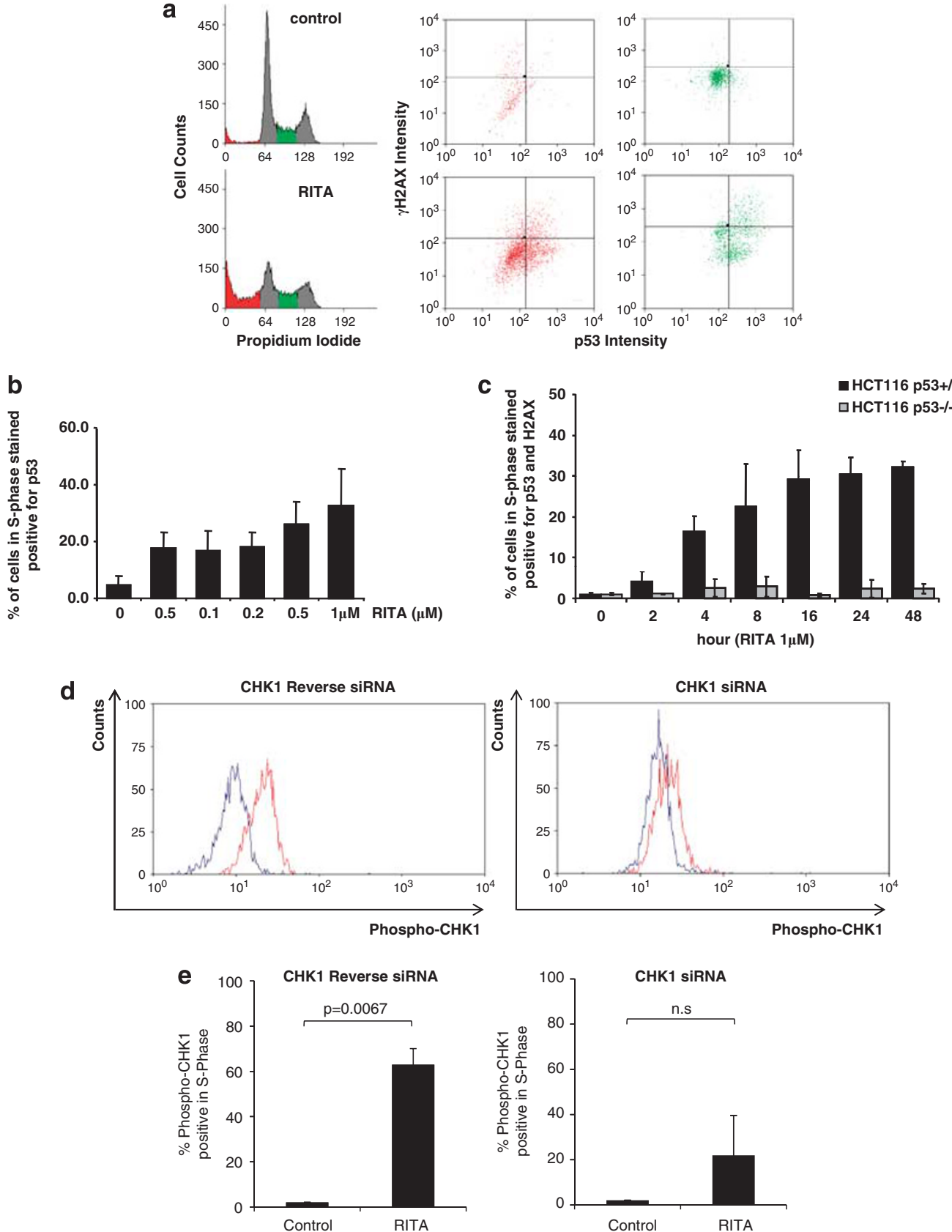

Figure 5 RITA induces p53 and phosphorylated CHK-1 and $\gamma \mathrm{H} 2 \mathrm{AX}$ proteins in S-phase. (a) Representative flow cytometric analysis plots of $\mathrm{p} 53^{+1+} \mathrm{HCT} 116 \mathrm{cells}$ treated with RITA $(500 \mathrm{nM})$ for $24 \mathrm{~h}$. Cells were stained for DNA content, using propidium iodide, p53 and phosphorylated-S139- $\gamma \mathrm{H} 2 \mathrm{AX}$ proteins. DNA profiles (graphs) were gated for cells in sub-G1 (red) and S-phases (green). Changes were quantified within the top right quadrant of each of dot plot. This quadrant indicates an increase in both p53 and phosphorylated-S139- $\gamma \mathrm{H} 2 \mathrm{AX}$ staining intensity. (b) $\mathrm{p5} 3^{+/+} \mathrm{HCT} 116$ cells were treated with RITA at the concentrations indicated for $24 \mathrm{~h}$ and harvested for flow cytometric analysis as described in a. Graph shows average percentage of cells in S-phase that stain positive for both p53 and phosphorylated-S139- $\gamma \mathrm{H} 2 \mathrm{AX}$ proteins. (c) p53 ${ }^{+1}$

${ }^{+} \mathrm{HCT} 116$ and $\mathrm{p5}^{-1-} \mathrm{HCT} 116$ cells were treated with RITA $(1 \mu \mathrm{M})$ for the indicated time and harvested for flow cytometric analysis as described in a. Graph shows average percentage of cells in S-phase that stain positive for both p53 and phosphorylated-S139- $\gamma \mathrm{H} 2 \mathrm{AX}$ proteins. Data have been averaged from three independent repeat experiments. (d and e) CHK-1 is phosphorylated in S-phase cells in response to RITA. p53 ${ }^{+/+}$HCT116 cells were transfected with either CHK-1 reverse siRNA, or CHK-1 targeted siRNA before RITA treatment ( $500 \mathrm{~nm}$ ) for $24 \mathrm{~h}$. (d) Propidium iodide FACS profiles were gated for the S-phase population of cells, and phosphorylated CHK-1 protein was quantified in the S-phase population (blue = control, red =RITA). The data is representative of two experiments. (e) Graphs show data described in $\mathbf{d}$ as the percentage $(\%)$ of cells in S-phase expressing phosphorylated CHK-1. n.s., not significant

induced by RITA, we knocked down CHK-1 using siRNA (Figure 5e). Interestingly, we found that CHK-1 knockdown had no significant effect on p53-dependent apoptotic responses induced by RITA (Figures $6 a$ and b), whereas the inhibition of caspase-3 activation by the inhibitor Z-DEVD-FMK blocked the increase in cells in sub-G1 (Figure 6a) and the increase in cleaved PARP (Figure 6b), but had no significant effect on phosphorylated CHK-1 
a
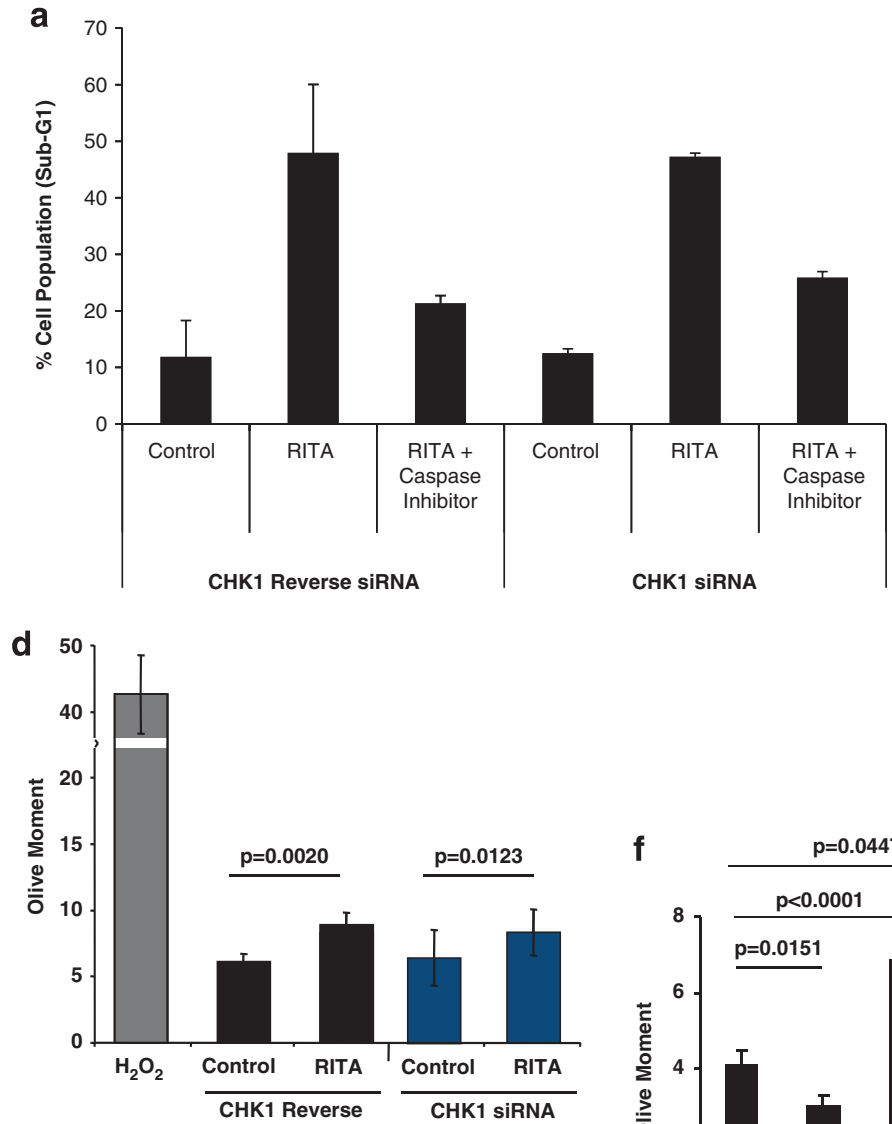

e

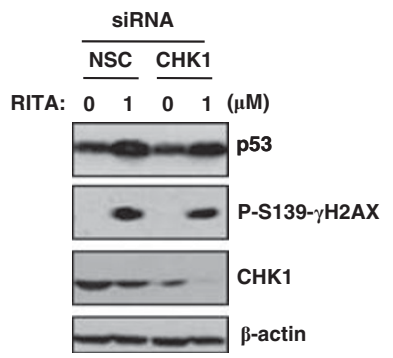

b

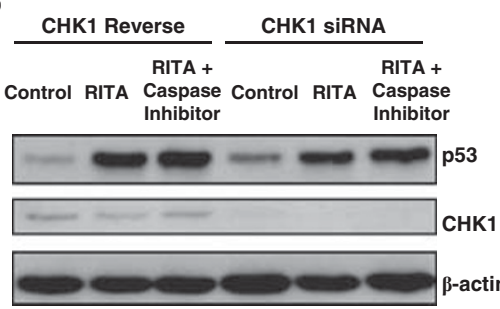

C

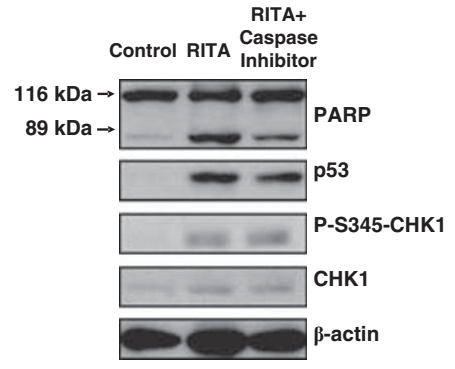

f $\quad \mathrm{p}=0.044$

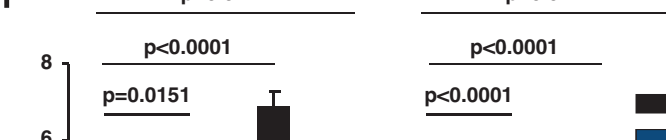

Control (0 hr) RITA (500nM) 1 hr

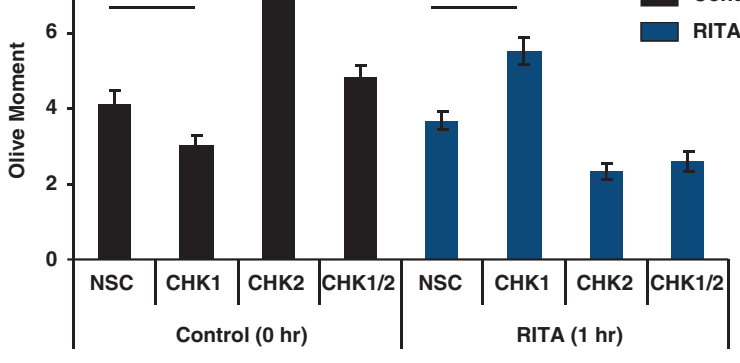

Figure 6 CHK-1 knockdown does not affect RITA-induced apoptosis but significantly enhances DNA damage in cells treated with RITA. (a and $\mathbf{b})$ p53 ${ }^{+/+}$HCT116 cells transiently transfected with a non-silencing control (NSC) siRNA duplex and siRNA to CHK-1 and treated with RITA (1 $\mu \mathrm{M})$ for $16 \mathrm{~h}$ in the presence and absence of the caspase-3 inhibitor Z-DEVD-FMK. Cells were harvested for flow cytometric analysis (a) or western analysis (b). (a) Graph shows the percentage of cells within sub-G1, as indicated. Data are representative of two independent experiments ( \pm S.D.). (b) Western blot analysis shows p53 and CHK-1 proteins. Actin was used as a loading control. (c) $5^{+1+} 3^{+1}$ HCT116 cells treated with RITA $(1 \mu \mathrm{M})$ for $16 \mathrm{~h}$ in the presence and absence of the caspase-3 inhibitor Z-DEVD-FMK. Western blot analysis shows PARP, p53, CHK-1 and phosphorylated-S139- $\gamma \mathrm{H} 2 \mathrm{AX}$ proteins. Actin was used as a loading control. (d) $\mathrm{p} 53^{+/+} \mathrm{HCT} 116$ cells transiently transfected with a NSC siRNA duplex and siRNA to CHK-1 and treated with RITA ( $500 \mathrm{nM}$ ) for $16 \mathrm{~h}$ and harvested for assessment of DNA damage using the Comet Score software (TriTek Corporation, Sumerduck, VA, USA). Graph shows mean olive moment ( $\sim 100$ comets/sample) for each condition as indicated. As a positive control for DNA damage, cells were treated with $\mathrm{H}_{2} \mathrm{O}_{2}(100 \mu \mathrm{M}$ for $20 \mathrm{~min}$ ). (e) In parallel with d, cells were harvested for western blot analysis. Western blots show p53, CHK-1 and phosphorylated-S139- $\gamma \mathrm{H} 2 \mathrm{AX}$ proteins. Actin was used as a

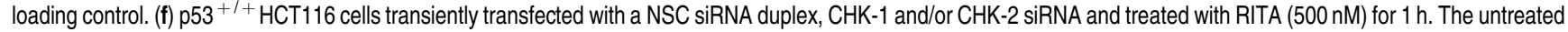
control samples were harvested at $0 \mathrm{~h}$. Cells were harvested for assessment of DNA damage using a comet assay, as described in $\mathbf{d}$. Graph shows mean olive moment $(\sim 100$ comets/sample) for each condition as indicated

induced by RITA (Figure 6c). CHK-1 has an important role in DNA repair $^{26}$ and is essential for the maintenance of genomic stability, ${ }^{27}$ particularly during DNA replication and replication fork progression. ${ }^{25,28-30}$ Thus, we next investigated whether CHK-1 knockdown affected the DNA damage response induced by RITA. We found that $\mathrm{CHK}-1$ knockdown had no significant effect on the small amount of accumulated DNA damage at $24 \mathrm{~h}$ of exposure to RITA
(Figure 6d), nor was there any significant effect on the $\gamma \mathrm{H} 2 \mathrm{AX}$ induced by RITA in CHK-1 knockdown cells at this time point (Figure 6e). Notably, a previous report has shown that phosphorylation at Ser345 on CHK-1 increases its turnover, and thereby reduces total $\mathrm{CHK}-1$ protein levels. ${ }^{31}$ Consistently, we found that total $\mathrm{CHK}-1$ protein levels decreased in the presence of RITA (Figure 6e). This effect was more dramatic in the presence of CHK-1 siRNA (Figure 6e). 
Finally, consistent with a role for $\mathrm{CHK}-1$ in DNA repair and maintenance, ${ }^{26,27}$ we found that $\mathrm{CHK}-1$ but not $\mathrm{CHK}-2$ knockdown significantly enhanced DNA damage induced at only $1 \mathrm{~h}$ of RITA treatment (Figure 6f), indicating that $\mathrm{CHK}-1$ is essential for maintaining DNA integrity upon short term exposure of cells to RITA. Consistently, we found that $\mathrm{CHK}-1$ phosphorylation was induced by RITA during this time frame (data not shown).

\section{Discussion}

HIF is upregulated in most human cancers due to changes in tumour microenvironmental stimuli and genetic abnormalities. ${ }^{1}$ Of particular interest, is the small-molecule approaches that have been taken recently to target the HIF pathway as a basis for the development of new therapeutics in the treatment of cancer. ${ }^{2,32}$ The p53 tumour suppressor protein is a potent negative regulator of HIF signalling in tumours. ${ }^{5}$ We have recently shown that small-molecule activator of p53, RITA, can mediate both antiangiogenic effects via blockade of the HIF pathway and elicit apoptosis in hypoxic tumour cells in vitro and in vivo. ${ }^{15}$ Intriguingly, unlike other p53-activating agents, RITA causes significant tumour cell apoptosis in normoxia and hypoxia $\left(1 \% \mathrm{O}_{2}\right)$, without eliciting either a measurable $\mathrm{G} 1$ and/or $\mathrm{G} 2$ arrest. ${ }^{15}$ Given that hypoxic tumour cells expressing high basal levels of HIF- $\alpha$ are usually resistant to killing by conventional radio and chemotherapeutic agents, in this study, we further investigated the mechanistic properties of RITA, with particular focus on exploring the DNA damage response.

Here, we found that RITA activated the canonical ATM/ATR DNA damage response pathway that leads to activation of $\mathrm{CHK}-1$ and $\mathrm{CHK}-2$ phosphorylation. Intriguingly, however, and confirming our recent findings, ${ }^{17}$ we found that the induction of phosphorylated $\mathrm{CHK}-1$ and $\gamma \mathrm{H} 2 \mathrm{AX}$ proteins observed in response to RITA was dependent on p53 status.

Previous studies have reported a p53-dependent DNA damage checkpoint. ${ }^{33,34}$ Activation of a p53-dependent S-phase DNA damage checkpoint occurs to delay DNA synthesis and to allow time to resolve a potential replication block. $^{33,35}$ Our earlier immunohistochemical analyses showed that RITA induced a pan-nuclear localisation of $\gamma \mathrm{H} 2 \mathrm{AX}$ opposed to localisation to discrete nuclear foci. ${ }^{17}$ This type of DNA damage response indicates potential stalling of the replication fork or is mediated during processing of bulky DNA lesions. ${ }^{22}$ Indeed, we found that RITA induced a p53dependent increase in replication fork number in a subpopulation of replicons. Notably, a decline in replication fork rate is known to be consistent with increased rates of local origin activation and higher replication fork densities, ${ }^{25}$ indicating that RITA stalled DNA replication elongation and affected replication fork rate.

Consistent with these observations, we found that treatment of cells with RITA also prolonged mid-late S-phase progression in p53-positive cells. Our findings indicate that RITA activates a p53-dependent checkpoint that may involve $\mathrm{CHK}-1$.

CHK-1 has been shown to have an important role in regulating DNA replication fork elongation and S-phase progression. ${ }^{25}$ In response to RITA, we found that the relative percentage of cells in S-phase expressing either p53 or $\gamma \mathrm{H} 2 \mathrm{AX}$ protein was increased compared with other cell cycle phases. Concurrently, we observed a significantly high percentage of cells in S-phase expressing phosphorylated CHK-1 upon RITA treatment. Activation of CHK-1 is crucial for eliciting replication checkpoints in response to DNA-damaging agents, providing protection to cells by allowing a slowing of S-phase progression, ${ }^{28,29}$ and appears to be critically involved in stabilising stalled replication forks. ${ }^{30}$ In addition, CHK-1 is important in DNA repair upon exposure to hydroxyurea. $^{26,36}$ Interestingly, we found that knockdown of CHK-1 by siRNA had only a minimal effect on the induction of p53-dependent apoptosis and $\gamma \mathrm{H} 2 \mathrm{AX}$ in response to RITA. However, knockdown of CHK-1 but not CHK-2 significantly increased DNA damage induced within only $1 \mathrm{~h}$ of RITA treatment, indicating that the activation of $\mathrm{CHK}-1$ mediated by RITA is important for maintaining DNA integrity.

RITA was originally identified using a cell-based screen ${ }^{12}$ and proposed to bind to the $\mathrm{N}$ terminus of p53 and induce $\mathrm{p53}$ stabilisation by disruption of the p53-HDM2 interaction. ${ }^{12}$ Other studies have shown that RITA can also cause proteinDNA and DNA-DNA intrastrand crosslinks, ${ }^{13,14}$ thereby suggesting that RITA may also act to stabilise p53 by interchelating with DNA. This latter possibility is supported by the observations that RITA activates the canonical DNA damage response. Of significance here is our observation that induction of CHK-1 phosphorylation by RITA is affected by p53 status. These findings suggest that RITA functions mechanistically both at the level of DNA and at the level of p53. Along with its role in G1/S- and G1/M-dependent cell cycle checkpoints, p53 has also been implicated in S-phase processes and DNA repair. Specifically, p53 is transported to sites of stalled DNA replication forks and binds to RAD51, ${ }^{37}$ thus p53 provides an S-phase-specific role that is independent of its known transcriptional activities. ${ }^{37}$ In addition, CHK-1 binds to and phosphorylates RAD51, providing a vital role in DNA repair upon exposure to hydroxyurea. ${ }^{36}$ Collectively, these events ensure that DNA integrity is maintained, and it may be that p53 itself provides an important role in a molecular sensor mechanism at the level of DNA.

In conclusion, our study highlights a novel role for p53 in the activation of a p53-dependent S-phase replication checkpoint that involves $\mathrm{CHK}-1$ and functions to protect the integrity of DNA. As we have previously shown that exposure of tumour cells to RITA leads to significant p53-dependent apoptosis in normoxia and hypoxia, ${ }^{15}$ it will be of particular interest to further examine the precise molecular mechanisms underlying this p53-dependent S-phase checkpoint in hypoxia.

\section{Materials and Methods}

Cell culture. All tumour cell lines were maintained in Dulbecco modified Eagle's medium. Medium was supplemented with $10 \%$ fetal calf serum purchased from Harlan (Oxford, UK), $100 \mathrm{IU} / \mathrm{ml}$ penicillin, $100 \mu \mathrm{g} / \mathrm{ml}$ streptomycin and $2 \mathrm{mM}$ glutamine (all purchased from Gibco/Life Technologies, Paisley, UK). The matched colorectal cell lines $p 53^{-1-} \mathrm{HCT} 116$ and $\mathrm{p} 53^{+/+} \mathrm{HCT} 116$ have been described previously. ${ }^{38}$ Human MCF-7 (breast carcinoma) and Saos-2 (osteosarcoma) cells were purchased from American Type Culture Collection (Manassass, VA, USA).

siRNA duplexes and transient transfection. The siRNA to $\mathrm{CHK}-1$ (5'-GGTGCCTATGGAGAAGTT-3') or CHK-2 (5'-CTTGAAGAGGTATCCGUGG-3') was obtained as a gel-purified annealed duplex from Dharmacon (Lafayette, CO, 
USA) and used at a final concentration of $25 \mathrm{nM}$, respectively. The non-silencing control siRNA duplex (5'-AATTCTCCGAACGTGTCACGT-3') was obtained from QIAGEN (Crawley, UK) and has been used by us previously. ${ }^{39}$ Transient transfections with siRNA duplexes were carried out using HiPerfect transfection reagent (QIAGEN) according to the manufacturer's instructions.

Antibodies. The HIF- $\alpha$ monoclonal antibody was purchased from BD Transduction Laboratories (Oxford, UK). The p53 monoclonal antibody (DO-1) was purchased from Calbiochem (Merck Biosciences, Nottingham, UK). The CHK1 monoclonal and CHK2 polyclonal antibodies were purchased from Santa Cruz Biotechnology (Santa Cruz, CA, USA). The p53 polyclonal antibody, polyclonal anti-phospho-S345-CHK1, polyclonal anti-phospho-T68-CHK2, monoclonal antiphospho-S15-p53, polyclonal anti-phospho-S20-p53 and polyclonal anti-phosphoS46P-p53 were all purchased from Cell Signaling Technologies (Danvers, MA, USA). The anti-phospho-S139- $\gamma \mathrm{H} 2 \mathrm{AX}$ monoclonal antibody was purchased from Upstate (Millipore, Newtownabbey, Northern Ireland).

Inductions and drug treatments. Physiological hypoxia was achieved by incubating cells in $1 \%$ oxygen, $5 \%$ carbon dioxide and $94 \%$ nitrogen in a LEEC (Nottingham, UK) dual gas incubator (GA-156). The hypoxic mimetic agent, deferoxamine mesylate (DFX), was used at a final concentration of $500 \mu \mathrm{M}$. RITA was obtained from the National Cancer Centre, Drug Therapeutic Program, Frederick, MD, USA (NSC-652287) and dissolved in dimethyl sulfoxide. Wortmannin (Sigma, Gillingham, UK) was used at a final concentration of $10 \mu \mathrm{M}$. The caspase-3 inhibitor Z-DEVD-FMK (Calbiochem) was used at $50 \mu \mathrm{M}$.

Western blot analysis. After treatment, cells were washed in ice-cold phosphate-buffered saline and lysed in $2 \times$ sample buffer $(125 \mathrm{mM}$ Tris $(\mathrm{pH} 6.8)$, $4 \%$ SDS, $0.01 \%$ bromophenol blue, $10 \% \beta$-mercaptoethanol, $10 \%$ glycerol). Alternatively, cells were harvested in NP-40 lysis buffer (100 mM Tris (pH 8.0), $100 \mathrm{mM} \mathrm{NaCl}_{2}, 1 \% \mathrm{NP}-40$ ) containing an EDTA-free protease inhibitor cocktail (Boehringer Mannheim-Roche Diagnostics Ltd, Burgess Hill, UK) to determine total protein concentration using a standard protein assay (Biorad, Hemel Hempstead, UK).

Flow cytometric analysis. Cell death was analysed by FACS using a Beckman Coulter Diagnostics machine (High Wycombe, UK). Briefly, total populations of cells, including floating and adherent cells, were fixed in $70 \%$ ethanol and stained with propidium iodide $(50 \mu \mathrm{g} / \mathrm{ml})$. Ribonuclease was added at $100 \mu \mathrm{g} / \mathrm{ml}$. The percentage of cells with sub-G1 DNA content was taken as a measurement of apoptosis.

Comet assay. The comet assay was performed using reagents from Trevigen (Gaithersburg, MD, USA) and according to the manufacturer's instructions. Cells were dosed for $24 \mathrm{~h}$ with RITA (at the concentrations indicated) in complete media. Following dosing, cells were harvested, mixed with low-melting agarose at $2 \times 10^{5}$ cells $/ \mathrm{ml}$ ( $\sim 1000$ cells/slide) and spread onto preprepared comet slides. Cells were lysed and incubated in alkaline buffer to select for single-strand DNA breaks. Slides were then electrophoresed using alkaline electrophoresis buffer at $4{ }^{\circ} \mathrm{C}, 18 \mathrm{v}$ and $300 \mathrm{~mA}$ for $40 \mathrm{~min}$. Following electrophoresis, slides were fixed, dried and stained using Sybr green (Trevigen). Comets were viewed using a Zeiss (Zeiss Ltd, Welwyn Garden City, Hertfordshire, UK) fluorescent microscope at $\times 20$ magnification, and images were captured over 20 fields of view for each slide using ImagePro software (Media Cybernetics Inc., Bethesda, MD, USA). The relative length and intensity of Sybr green-stained nuclei (comets) were proportional to DNA damage in individual nuclei. This was quantified using an algorithm for Olive tail moment on the CometScore software (TriTek Corporation, Sumerduck, VA, USA). At least 100 comets were analysed for each treatment. A 20-min dose of $100 \mu \mathrm{M}$ hydrogen peroxide at $4{ }^{\circ} \mathrm{C}$ was used as a positive control for DNA damage.

DNA fibre assay. Replication tracks were labelled in culture medium containing $25 \mu \mathrm{M}$ BrdU. RITA (500 nM) was added $16 \mathrm{~h}$ before each experiment. DNA fibre spreads were prepared, as previously described. ${ }^{24}$ BrdU-labelled tracks were detected with BrdU anti-sheep antibody (Biodesign, Lewisville, TX, USA; M20105S 1:1000 dilution; $1 \mathrm{~h}$ at $20^{\circ} \mathrm{C}$ ) using either Cy3- or AlexaFluor-488-conjugated donkey anti-sheep secondary antibody (Invitrogen Ltd, Paisley, UK). Fibres were examined using a Zeiss LSM 510 confocal microscope using a $\times 100$ (1.4NA) lens, labelled tracks measured using the LSM software (Zeiss Ltd) (white bars on individual images show examples of measurements recorded) and converted to kbp using a conversion factor of $1 \mu \mathrm{m}=2.59 \mathrm{kbp}$. Measurements were recorded in randomly selected fields (selected at low power) from dispersed, untangled areas of the DNA spread. As the analysis of single, unbroken fibres is a key, routine quality control for spreading of different cell types under different experimental conditions was performed using direct DNA labelling with YOYO. ${ }^{23}$ For the S-phase analysis, cells were grown on microscope coverslips and pulsed labelled for $20 \mathrm{~min}$ with $25 \mu \mathrm{M}$ BrdU. RITA $(500 \mathrm{nM})$ was added $16 \mathrm{~h}$ before each experiment. The cells were fixed using $4 \% \mathrm{PF}$, and BrdU detected as described above and previously. ${ }^{25}$

\section{Conflict of Interest}

The authors declare no conflict of interest.

Acknowledgements. We thank Anna EO Fisher, Nadia Lovegrove and Keith W Caldecott (Genome Damage and Stability Centre, University of Sussex, Falmer, Brighton, UK) for their initial input with comet assays. We thank David Gillespie (The Beatson Institute for Cancer Research, Glasgow, UK) for helpful discussions. We also thank Bert Vogelstein (Johns Hopkins School of Medicine, Baltimore, MD, USA) for the $\mathrm{p} 53^{-/-}$HCT116 and $p 53^{+1+}$ HCT116 cells. ${ }^{38}$ Finally, we thank all other members of the Ashcroft lab for their input. AA and JY were funded by the Cancer Research UK studentships C7358/A8020 and C7358/A4420, respectively. AM-M and DAJ were funded by a BBSRC grant (reference BBS/B/06091).

1. Majmundar AJ, Wong WJ, Simon MC. Hypoxia-inducible factors and the response to hypoxic stress. Mol Cell 2010; 40: 294-309.

2. Poon E, Harris AL, Ashcroft M. Targeting the hypoxia-inducible factor (HIF) pathway in cancer. Expert Rev Mol Med 2009; 11: e26.

3. Graeber TG, Osmanian C, Jacks T, Housman DE, Koch CJ, Lowe SW et al. Hypoxiamediated selection of cells with diminished apoptotic potential in solid tumours. Nature 1996; 379: 88-91.

4. Stempien-Otero A, Karsan A, Cornejo CJ, Xiang H, Eunson T, Morrison RS et al. Mechanisms of hypoxia-induced endothelial cell death. Role of p53 in apoptosis. J Biol Chem 1999; 274: 8039-8045.

5. Ravi R, Mookerjee B, Bhujwalla ZM, Sutter CH, Artemov D, Zeng $Q$ et al. Regulation of tumor angiogenesis by p53-induced degradation of hypoxia-inducible factor 1alpha. Genes Dev 2000; 14: 34-44.

6. Kaluzova M, Kaluz S, Lerman MI, Stanbridge EJ. DNA damage is a prerequisite for p53mediated proteasomal degradation of HIF-1alpha in hypoxic cells and downregulation of the hypoxia marker carbonic anhydrase IX. Mol Cell Biol 2004; 24: 5757-5766.

7. Salnikow K, Costa M, Figg WD, Blagosklonny MV. Hyperinducibility of hypoxia-responsive genes without p53/p21-dependent checkpoint in aggressive prostate cancer. Cancer Res 2000; 60: 5630-5634.

8. Foster BA, Coffey HA, Morin MJ, Rastinejad F. Pharmacological rescue of mutant p53 conformation and function. Science 1999; 286: 2507-2510.

9. Bykov VJ, Wiman KG. Novel cancer therapy by reactivation of the p53 apoptosis pathway. Ann Med 2003; 35: 458-465.

10. Vassilev LT, Vu BT, Graves B, Carvajal D, Podlaski F, Filipovic Z et al. In vivo activation of the p53 pathway by small-molecule antagonists of MDM2. Science 2004; 303: 844-848.

11. Tovar C, Rosinski J, Filipovic Z, Higgins B, Kolinsky K, Hilton $\mathrm{H}$ et al. Small-molecule MDM2 antagonists reveal aberrant p53 signaling in cancer: implications for therapy. Proc Natl Acad Sci USA 2006; 103: 1888-1893.

12. Issaeva N, Bozko P, Enge M, Protopopova M, Verhoef LG, Masucci M et al. 2004 Small molecule RITA binds to p53, blocks p53-HDM-2 interaction and activates p53 function in tumors. Nat Med 10: 1321-1328.

13. Rivera MI, Stinson SF, Vistica DT, Jorden JL, Kenney S, Sausville EA. Selective toxicity of the tricyclic thiophene NSC 652287 in renal carcinoma cell lines: differential accumulation and metabolism. Biochem Pharmacol 1999; 57: 1283-1295.

14. Nieves-Neira W, Rivera MI, Kohlhagen G, Hursey ML, Pourquier P, Sausville EA et al. DNA protein cross-links produced by NSC 652287, a novel thiophene derivative active against human renal cancer cells. Mol Pharmacol 1999; 56: 478-484.

15. Yang J, Ahmed A, Poon E, Perusinghe N, de Haven Brandon A, Box G et al. Smallmolecule activation of p53 blocks hypoxia-inducible factor 1alpha and vascular endothelial growth factor expression in vivo and leads to tumor cell apoptosis in normoxia and hypoxia. Mol Cell Biol 2009; 29: 2243-2253.

16. Enge M, Bao W, Hedstrom E, Jackson SP, Moumen A, Selivanova G. MDM2-dependent downregulation of $\mathrm{p} 21$ and hnRNP $\mathrm{K}$ provides a switch between apoptosis and growth arrest induced by pharmacologically activated p53. Cancer Cell 2009; 15: 171-183.

17. Yang J, Ahmed A, Ashcroft M. Activation of a unique p53-dependent DNA damage response. Cell Cycle 2009; 8: 1630-1632.

18. Kao J, Milano MT, Javaheri A, Garofalo MC, Chmura SJ, Weichselbaum RR et al. gamma$\mathrm{H} 2 \mathrm{AX}$ as a therapeutic target for improving the efficacy of radiation therapy. Curr Cancer Drug Targets 2006; 6: 197-205. 
19. Ashcroft M, Kubbutat MH, Vousden $\mathrm{KH}$. Regulation of p53 function and stability by phosphorylation. Mol Cell Biol 1999; 19: 1751-1758.

20. Bradbury JM, Jackson SP. ATM and ATR. Curr Biol 2003; 13: R468.

21. Chehab NH, Malikzay A, Stavridi ES, Halazonetis TD. Phosphorylation of Ser-20 mediates stabilization of human p53 in response to DNA damage. Proc Natl Acad Sci USA 1999; 96 : 13777-13782.

22. Marti TM, Hefner E, Feeney L, Natale V, Cleaver JE. H2AX phosphorylation within the G1 phase after UV irradiation depends on nucleotide excision repair and not DNA doublestrand breaks. Proc Natl Acad Sci USA 2006; 103: 9891-9896.

23. Merrick CJ, Jackson D, Diffley JF. Visualization of altered replication dynamics after DNA damage in human cells. J Biol Chem 2004; 279: 20067-20075.

24. Jackson DA, Pombo A. Replicon clusters are stable units of chromosome structure: evidence that nuclear organization contributes to the efficient activation and propagation of S phase in human cells. J Cell Biol 1998; 140: 1285-1295.

25. Maya-Mendoza A, Petermann E, Gillespie DA, Caldecott KW, Jackson DA. Chk1 regulates the density of active replication origins during the vertebrate S phase. EMBO J 2007; 26 2719-2731.

26. Niida H, Murata K, Shimada M, Ogawa K, Ohta K, Suzuki K et al. Cooperative functions of Chk1 and Chk2 reduce tumour susceptibility in vivo. Embo J 2010; 29: 3558-3570.

27. Paulsen RD, Cimprich KA. The ATR pathway: fine-tuning the fork. DNA Repair (Amst) 2007; 6: 953-966.

28. Robinson HM, Jones R, Walker M, Zachos G, Brown R, Cassidy J et al. Chk1-dependent slowing of S-phase progression protects DT40 B-lymphoma cells against killing by the nucleoside analogue 5-fluorouracil. Oncogene 2006; 25: 5359-5369.

29. Petermann E, Maya-Mendoza A, Zachos G, Gillespie DA, Jackson DA, Caldecott KW. Chk1 requirement for high global rates of replication fork progression during normal vertebrate S phase. Mol Cell Biol 2006; 26: 3319-3326.

30. Petermann E, Woodcock M, Helleday T. Chk1 promotes replication fork progression by controlling replication initiation. Proc Natl Acad Sci USA 2010; 107: 16090-16095.

31. Collis SJ, Barber LJ, Clark AJ, Martin JS, Ward JD, Boulton SJ. HCLK2 is essential for the mammalian S-phase checkpoint and impacts on Chk1 stability. Nat Cell Biol 2007; 9: 391-401.
32. Semenza GL. Defining the role of hypoxia-inducible factor 1 in cancer biology and therapeutics. Oncogene 2009; 29: 625-634.

33. Shimura T, Toyoshima M, Adiga SK, Kunoh T, Nagai H, Shimizu N et al. Suppression of replication fork progression in low-dose-specific p53-dependent S-phase DNA damage checkpoint. Oncogene 2006; 25: 5921-5932.

34. Agarwal ML, Agarwal A, Taylor WR, Chernova O, Sharma Y, Stark GR. A p53-dependent S-phase checkpoint helps to protect cells from DNA damage in response to starvation for pyrimidine nucleotides. Proc Natl Acad Sci USA 1998; 95: 14775-14780.

35. Vaziri C, Saxena S, Jeon Y, Lee C, Murata K, Machida Y et al. A p53-dependent checkpoint pathway prevents rereplication. Mol Cell 2003; 11: 997-1008.

36. Sorensen CS, Hansen LT, Dziegielewski J, Syljuasen RG, Lundin C, Bartek J et al. The cell-cycle checkpoint kinase Chk1 is required for mammalian homologous recombination repair. Nat Cell Biol 2005; 7: 195-201.

37. Sengupta S, Linke SP, Pedeux R, Yang Q, Farnsworth J, Garfield SH et al. BLM helicasedependent transport of p53 to sites of stalled DNA replication forks modulates homologous recombination. EMBO J 2003; 22: 1210-1222.

38. Bunz F, Dutriaux A, Lengauer C, Waldman T, Zhou S, Brown JP et al. Requirement for p53 and p21 to sustain G2 arrest after DNA damage. Science 1998; 282: 1497-1501.

39. Carroll VA, Ashcroft M. Role of hypoxia-inducible factor (HIF)-1alpha versus HIF-2alpha in the regulation of HIF target genes in response to hypoxia, insulin-like growth factor-I, or loss of von Hippel-Lindau function: implications for targeting the HIF pathway. Cancer Res 2006; 66: 6264-6270.

Cell Death and Disease is an open-access journal published by Nature Publishing Group. This work is licensed under the Creative Commons Attribution-Noncommercial-No Derivative Works 3.0 Unported License. To view a copy of this license, visit http://creativecommons.org/licenses/by-nc-nd/3.0/ 